\title{
Effet comparé de l'enfouissement de deux légumineuses fertilisées au molybdène sur la croissance et le rendement de Brachiaria ruziziensis à différentes périodes de fauche dans l'Ouest Cameroun
}

\author{
Arsène V. MBOKO ${ }^{1}$, Fernand TENDONKENG ${ }^{2}$, Férence N. E. MATUMUINI ${ }^{1}$, \\ Gilbert T. ZOUGOU ${ }^{1}$, Emile MIEGOUE ${ }^{2}$, Benoît BOUKILA ${ }^{1}$ et Etienne T. PAMO ${ }^{2 *}$ \\ ${ }^{1}$ Institut National Supérieur d'Agronomie et de Biotechnologie (INSAB), \\ Université des Sciences et Techniques de Masuku, B.P. 941 Franceville, Gabon. \\ ${ }^{2}$ Laboratoire de Nutrition Animale, Département des Productions Animales, FASA, \\ Université de Dschang. B.P. 222 Dschang, Cameroun. \\ *Auteur correspondant; E-mail : pamo_te@yahoo.fr
}

\section{RESUME}

Un essai sur l'effet comparé de l'enfouissement d'Arachis glabrata et de Desmodium intortum fertilisés au molybdène sur la croissance et le rendement de Brachiaria ruziziensis a été conduit à la Ferme d'Application et de Recherche de l'Université de Dschang. Un dispositif factoriel $4 \times 2$ (4 niveaux de fertilisation au molybdène, $0 ; 0,75 ; 1,52,25 \mathrm{~kg} / \mathrm{ha}$ et 2 légumineuses) en 2 répétitions/blocs et disposé en 3 blocs de16 parcelles élémentaires chacun a été utilisé sur des parcelles de $6 \mathrm{~m}^{2}$. Neuf mois après la mise en place des légumineuses, elles ont été fauchées et enfouies entièrement. Des éclats de souche de Brachiaria ruziziensis ont été ensuite cultivés à une densité de 11 plants $/ \mathrm{m}^{2}$ sur toutes les parcelles. Tous les 45 jours, la biomasse et la taille des plantes étaient évaluées sur une surface utile de $2 \mathrm{~m}^{2}$ par niveau de fertilisation. Les résultats ont montré que : l'enfouissement des légumineuses a influencé de manière variable la taille du fourrage. La taille la plus élevée $(83,4 \mathrm{~cm})$ a été obtenue avec le précédent Desmodium intortum fertilisé aux doses de 0 et de $0,75 \mathrm{~kg}$ de $\mathrm{Mo} / \mathrm{ha}$, à la première fréquence de fauche. La biomasse sèche de Brachiaria ruziziensis a été peu influencée par l'enfouissement des légumineuses. La biomasse la plus élevée $(25 \mathrm{t} \mathrm{MS/ha)}$ a été obtenue avec le précédent Desmodium intortum fertilisé à $0,75 \mathrm{~kg}$ de Mo/ha. En somme, l'enfouissement de Desmodium intortum fertilisé avec la dose de $0,75 \mathrm{~kg}$ de $\mathrm{Mo} / \mathrm{ha}$, a permis d'obtenir la taille et la biomasse sèche les plus élevées à la première période de fauche, sans qu'aucune différence significative $(p>0,05)$ n'ait été observé entre les traitements.

(ㅇ) 2013 International Formulae Group. All rights reserved.

Mots clés: Arachis glabrata, biomasse, Desmodium intortum, taille.

\section{INTRODUCTION}

Dans le souci de réduire les coûts de production, résoudre le problème de l'indisponibilité de l'engrais sur le marché et éviter la pollution de l'environnement par l'utilisation d'engrais chimique, les producteurs s'orientent de plus en plus vers des ressources énergétiques naturelles 
agricoles (Brkić et al., 2004). Ainsi, l'utilisation du fumier de ferme, du compost, des associations culturales (légumineusesgraminées), la rotation de culture avec enfouissement des légumineuses etc. constituent dans cette optique, des atouts indéniables. Ainsi, les légumineuses herbacées, vivaces ou subligneuses telles que Abrus precatorius, Calopogonium mucunoides, Centrosema pubescens, Clitoria ternatea, Crotalaria goreensis etc. semblent être des alternatives intéressantes, du fait de leur capacité d'enrichissement rapide des sols par la fixation d'azote atmosphérique et la production abondante de biomasse végétale (Akedrin et al., 2010). Les légumineuses de couvertures comme Mucuna pruriens, Pueraria phaseoloïdes, Lablab purpureus..., permettent une accumulation assez importante d'azote allant de 61 à $650 \mathrm{~kg} / \mathrm{ha}$ dans les jachères améliorées (Segda et al., 1998). De même, en injectant au sol de l'azote organique par le biais de l'incorporation de la matière organique comme engrais vert, elles augmentent également l'azote minéralisable du sol (Le Soudeur et Labreuche, 2007). Toutefois, les effets des engrais verts sur l'activité biologique et la minéralisation de l'azote dans le sol dépendent principalement de leur composition chimique, notamment de leur concentration en azote et de leur rapport C/N (Frankenberger et abdelmagid, 1985 ; Janzen et al., 1990 ; Mahler et Hemanda 1993). Mais, la déficience en éléments comme le molybdène, ou le bore et le zinc peut compromettre la croissance et la production des légumineuses (Roberge et Toutain, 1999). Le molybdène est un constituant des enzymes impliquées dans la fixation de l'azote gazeux par les Rhizobiums des légumineuses et la réduction des nitrates. Ainsi, l'ion ammonium $\left(\mathrm{NH}_{4}{ }^{+}\right)$provenant de cette réduction est incorporé dans les squelettes carbonés pour former les acides aminés par le processus d'assimilation de l'azote (Morot-Gaudry, 1997).

Bien que l'utilisation des légumineuses en tête de rotation de culture avec ou sans enfouissement des biomasses constitue une pratique courante en culture vivrière en zone rurale, peu d'informations existent sur la quantité d'azote que cette pratique peut fournir. Aussi, les effets bénéfiques d'une légumineuse dans une rotation de culture avec enfouissement en système d'exploitation fourragère non fertilisé sont peu connus et appréciés et les informations non disponibles. L'objectif de cette étude est donc de contribuer à l'amélioration des connaissances sur l'effet de l'enfouissement d'Arachis glabrata et de Desmodium intortum fertilisés au molybdène sur la croissance et la production de Brachiaria ruziziensis.

\section{MATERIEL ET METHODES}

\section{Zone d'étude}

L'étude a été conduite à la Ferme d'Application et de Recherche (FAR) de l'Université de Dschang entre mars 2010 et Novembre 2012. La FAR est située en région Soudano-guinéenne du Cameroun à 05²0' latitude Nord et $10^{\circ} 03^{\prime}$ longitude Est et à une altitude moyenne de $1410 \mathrm{~m}$. Le climat de la région est équatorial de type Camerounien, modifié par l'altitude. Les températures oscillent entre $10{ }^{\circ} \mathrm{C}$ (juillet-août) et $25{ }^{\circ} \mathrm{C}$ (février), avec une insolation annuelle de 1800 heures et une humidité relative variant entre 40 - 97\%. Les précipitations varient entre 1500 et $2000 \mathrm{~mm}$ par an. La saison sèche va de mi-novembre à mi-mars, et la saison des pluies, correspondant à la principale période de culture de mi-mars à mi-novembre. La topographie $\mathrm{du}$ site expérimental est légèrement pentue, sur oxisol de profondeur élevée (plus de $5 \mathrm{~m}$ ) et sous pression humaine à travers la pratique des cultures vivrières annuelles. La végétation de cette région est une savane arbustive avec par endroit des forêts galeries.

\section{Dispositif expérimental}

Le dispositif expérimental utilisé était un plan factoriel $4 \times 2$ en deux répétitions/bloc et disposé en trois blocs de seize parcelles élémentaires chacun (soit 48 parcelles au 
total). Ce dispositif comportait quatre niveaux de fertilisation au molybdène sous forme de molybdate d'ammonium $(0 ; 0,75 ; 1,5$ et 2,25 $\mathrm{kg} / \mathrm{ha}$ ) et deux légumineuses (Arachis glabrata et Desmodium intortum), implantées sur des parcelles de $6 \mathrm{~m}^{2}(3 \times 2 \mathrm{~m})$ pour un total utilisé de 48 parcelles expérimentales. Pour des raisons d'homogénéité des conditions pédologiques, d'arrosage, de température et d'éclairement, les deux légumineuses, ont été aléatoirement soumis aux quatre traitements, dans chaque bloc. Les blocs étaient séparés les uns des autres par des allées de $1 \mathrm{~m}$. Les interlignes des parcelles élémentaires, au sein d'un même bloc, étaient de $0,5 \mathrm{~m}$.

\section{Préparation du sol, mise en place des plants et fertilisation}

L'essai a commencé par la préparation manuelle des parcelles et des lits de semence. Des touffes d'Arachis glabrata et de Desmodium intortum ont été prélevées sur les parcours de la FAR. Elles ont été habillées à l'aide de ciseaux. Soixante six talles de un à deux rejets obtenues par division manuelle ont été repiquées sur chaque parcelle, à $4 \mathrm{~cm}$ de profondeur, avec un écartement de $25 \mathrm{~cm}$ x 25 $\mathrm{cm}$.

Des engrais phosphaté et potassique sous forme de super phosphate simple (100 $\mathrm{kg} / \mathrm{ha}$ ) à $21 \%$ et de chlorure de potassium (35 $\mathrm{kg} / \mathrm{ha}$ ) ont été appliqués comme engrais de fond, sur l'ensemble des parcelles expérimentales.

Un mois après la mise en place des éclats de souches des légumineuses, un désherbage manuel des parcelles, des interlignes et des allées entre les parcelles a été réalisé. Chaque parcelle a été fertilisée au molybdène en une seule fois selon le plan expérimental. Afin de s'assurer d'une homogénéité dans l'application des quantités très faibles de molybdène, chaque dose a été dissoute dans de l'eau de façon à apporter exactement $10 \mathrm{ml}$ de solution au pied de chaque plante, ce qui correspondait à $0 ; 6,81$; 13,6 et 20,4 mg de Mo/pieds respectivement pour les doses de $0 ; 0,75 ; 1,50$ et $2,25 \mathrm{~kg}$ de $\mathrm{Mo} / \mathrm{ha}$.

Neuf mois après la mise en place d'Arachis glabrata et de Desmodium intortum, et après l'accomplissement de leur cycle végétatif, les plantes ont été fauchés à une hauteur de $20 \mathrm{~cm}$ du sol sur une surface utile de $2 \mathrm{~m}^{2}$, pour l'évaluation de la biomasse. Un échantillon représentatif de 500 $\mathrm{g}$ de la biomasse des légumineuses a été prélevé par niveau de fertilisation au molybdène en vue de l'évaluation de l'azote et du carbone suivant la méthode décrite par Pauwels et al. (1992) pour la détermination du rapport $\mathrm{C} / \mathrm{N}$ des légumineuses avant leur enfouissement. Les parcelles ont été ensuite fauchées entièrement. Les biomasses végétales préalablement hachées ont été manuellement enfouies dans leurs parcelles respectives à une profondeur de $20 \mathrm{~cm}$ dans le sol.

Deux mois après l'enfouissement, un labour léger avec des houes suivi de la préparation des lits de semence ont été effectués sur l'ensemble des parcelles expérimentales aux précédentes légumineuses avant la mise en place des éclats de souche de Brachiaria ruziziensis.

Des touffes de Brachiaria ruziziensis ont été prélevées sur les parcours de la FAR et habillées à l'aide de ciseaux. Soixante six talles de un à deux rejets obtenues par division manuelle de ces touffes ont ensuite été repiquées sur l'ensemble des parcelles à $4 \mathrm{~cm}$ de profondeur et suivant un écartement de 25 $\mathrm{cm} \times 25 \mathrm{~cm}$. Deux mois après la plantation des éclats de souche, une coupe de régularisation a été effectuée à $20 \mathrm{~cm}$ au dessus du sol, et les parcelles n'ont reçu aucune autre fertilisation.

\section{Evaluation de la croissance de Brachiaria ruziziensis}

A chaque période de fauche, vingt plantes étaient prélevées au hasard dans chaque parcelle, pour un total de 120 plantes (20 plantes x 3 répétitions par niveau de fertilisation et par légumineuse enfouie). La 
hauteur de plant collecté était alors évaluée à l'aide d'un mètre ruban.

\section{Evaluation de la biomasse de Brachiaria ruziziensis}

Pour chaque période de fauche, toutes les parcelles ont été fauchées tous les quarante cinq (45) jours. Pendant la fauche et pour éviter les effets de bordure, Brachiaria ruziziensis a été coupé au centre de la planche sur une superficie utile de $2 \mathrm{~m}^{2}$ à $5 \mathrm{~cm}$ du sol (Tendonkeng et al., 2009). Le fourrage récolté sur cette superficie a été ensuite pesé. Un échantillon représentatif de $0,5 \mathrm{~kg}$ de Brachiaria ruziziensis a été prélevé à chaque période de fauche par niveau de fertilisation au molybdène et par légumineuse enfouie, puis séché dans une étuve ventilée à $60{ }^{\circ} \mathrm{C}$ jusqu'à poids constant pour la détermination de la matière sèche et le rendement ( $\mathrm{MS} / \mathrm{ha})$.

\section{Analyses statistiques}

Les résultats relatifs à l'étude comparée de la hauteur et de la biomasse (t MS/ha) produite de Brachiaria ruziziensis, en fonction de l'enfouissement d'Arachis glabrata et de Desmodium intortum fertilisés au molybdène à différentes périodes de fauche ont été soumis à une analyse de la variance (ANOVA) à mesures répétées. Le logiciel utilisé pour traiter les données était GenStat 9.2. Lorsque les effets des facteurs de variations étaient significatifs, les moyennes ont été séparées par le test de la ppds au seuil de signification de $5 \%$.

\section{RESULTATS}

Effet de la fertilisation au molybdène sur les biomasses (t MS/ha) d'Arachis glabrata et de Desmodium intortum

La fertilisation au molybdène n'a eu aucun effet significatif $(\mathrm{p}>0,05)$ sur les biomasses de $A$. glabrata et de $D$. intortum (Figure 1). La biomasse la plus élevée de $A$. glabrata $(9,21$ t MS/ha) a été obtenue dans les parcelles non fertilisées, alors que celle de $D$. intortum (17,81 t MS/ha) a été obtenue sur les parcelles fertilisées à $2,25 \mathrm{~kg}$ de $\mathrm{Mo} / \mathrm{ha}$.
Avec le niveau $0 \mathrm{~kg}$ de Mo/ha, les biomasses des deux légumineuses ont été comparables $(\mathrm{p}>0,05)$. Par contre, les biomasses de $D$. intortum obtenues avec les niveaux $75,1,5$ et $2,25 \mathrm{~kg}$ de $\mathrm{Mo} / \mathrm{ha}$, ont été significativement $(\mathrm{p}<0,05)$ plus élevées que celles de A. glabrata.

Effet de la fertilisation au molybdène sur les rapports carbone/azote (C/N) d'Arachis glabrata et de Desmodium intortum

Le rapport $\mathrm{C} / \mathrm{N}$ de $A$. glabrata a augmenté avec le niveau de fertilisation jusqu'à $1,50 \mathrm{~kg}$ de $\mathrm{Mo} / \mathrm{ha}$, tandis que celui de D. intortum le plus élevé $(18,2)$ a été obtenu avec la fertilisation à $0,75 \mathrm{~kg}$ de $\mathrm{Mo} / \mathrm{ha}$ (Figure 2). En dehors de la dose de $1,50 \mathrm{~kg}$ de $\mathrm{Mo} / \mathrm{ha}$, où le rapport $\mathrm{C} / \mathrm{N}$ de $A$. glabrata a été plus élevé $(16,4)$, les rapports $\mathrm{C} / \mathrm{N}$ obtenus avec $D$. intortum ont été les plus élevés avec les autres niveaux de fertilisation. Cependant, aucune différence significative $(p>0,05)$ n'a été observée entre les rapports des deux légumineuses quel que soit le niveau de fertilisation au Molybdène.

Effet de l'enfouissement d'Arachis glabrata fertilisé au molybdène sur la taille $(\mathrm{cm})$ des plantes de Brachiaria ruziziensis à différentes périodes de fauche

L'évolution de la taille $(\mathrm{cm})$ des plantes de $B$. ruziziensis obtenue avec le précédent $A$. glabrata à différentes périodes de fauche montre que l'enfouissement de cette légumineuse fertilisée au molybdène n'a pas significativement $(\mathrm{p}>0,05)$ influencé les tailles des plantes de B. ruziziensis (Tableau 1). La taille la plus élevée des plantes obtenues à la première fauche, et la taille moyenne des plantes à l'issu des cinq périodes de fauche étant de $42 \mathrm{~cm}$.

Effet de l'enfouissement d'Arachis glabrata fertilisé au molybdène sur la biomasse de Brachiaria ruziziensis à différentes périodes de fauche

L'incorporation au sol de A. glabrata a relativement influencé la biomasse de $B$. ruziziensis. A la deuxième et troisième 
période de fauche, les biomasses obtenues ont présenté des effets significatifs en fonction des niveaux de fertilisation au molybdène (Tableau 2).

Les valeurs obtenues avec les doses de $0 ; 1,50$ et $2,25 \mathrm{~kg}$ de $\mathrm{Mo} / \mathrm{ha}$ à la deuxième période de fauche ont été comparables $(p>0,05)$ et significativement $(p<0,05)$ plus faibles que celle obtenue avec la dose de 0,75 $\mathrm{kg}$ de $\mathrm{Mo} / \mathrm{ha}$. A la troisième période de fauche, la biomasse obtenue avec la dose de $1,50 \mathrm{~kg}$ de $\mathrm{Mo} / \mathrm{ha}$ a été comparable $(\mathrm{p}>0,05)$ à celles obtenues avec les doses de 0 et $0,75 \mathrm{~kg}$ de Mo/ha et significativement $(\mathrm{p}<0,05)$ plus élevée (4,62 t MS/ha) que celle obtenue avec la dose de $2,25 \mathrm{~kg}$ de $\mathrm{Mo} / \mathrm{ha}$. De manière générale, la fertilisation à la dose de $0,75 \mathrm{~kg}$ de Mo/ha a permis d'obtenir la biomasse la plus élevée (39,42 t MS/ha) pour l'ensemble de la période de l'essai.

Effet de l'enfouissement de Desmodium intortum fertilisé au molybdène sur la taille (cm) des plantes de Brachiaria ruziziensis à différentes périodes de fauche

L'enfouissement de $D$. intortum a relativement influencé la taille de $B$. ruziziensis en fonction des doses de fertilisation de Mo aux différentes périodes de fauche (Tableau 3). En dehors de la troisième période où les plantes sous précédent $D$. intortum fertilisées à $1,50 \mathrm{~kg}$ de $\mathrm{Mo} / \mathrm{ha}$ ont présenté une taille significativement plus faible, la fertilisation n'a pas influencé de manière significative $(p>0,05)$ les tailles des plantes aux autres périodes de fauche.

En moyenne, les tailles des plantes ont été comparables ( $p>0,05)$, pour l'ensemble de l'essai quel que soit le niveau de fertilisation au molybdène et la taille des plantes des parcelles non fertilisées a été plus élevée.

Effet de l'enfouissement de Desmodium intortum fertilisé au molybdène sur la biomasse de Brachiaria ruziziensis à différentes périodes de fauche

La biomasse de B. ruziziensis obtenue avec le précédent $D$. intortum à différentes périodes de fauche montre qu'elle n'a pas été significativement influencée $(p>0,05)$ par les niveaux de fertilisation au molybdène (Tableau 4). Toutefois, la biomasse la plus élevée (44,2 t MS/ha), pour l'ensemble de l'essai, a été obtenue avec la fertilisation à la dose de $0,75 \mathrm{~kg}$ de $\mathrm{Mo} / \mathrm{ha}$.

Effet comparé de l'enfouissement d'Arachis glabrata et de Desmodium intortum fertilisés au molybdène sur la taille de Brachiaria ruziziensis à différentes périodes de fauche

Les tailles de B. ruziziensis obtenues avec les précédents culturaux en fonction des doses de molybdène n'ont été significativement $(\mathrm{p}<0,05)$ influencées qu'à la troisième période de fauche (Figure 2). A cette période de fauche, le précédent $D$. intortum a induit une taille de $B$. ruziziensis significativement $(\mathrm{p}<0,05)$ la plus élevée avec la dose de $0,75 \mathrm{~kg}$ de Mo/ha. Avec la dose de $1,50 \mathrm{~kg}$ de $\mathrm{Mo} / \mathrm{ha}$, c'est l'effet contraire qui a été observé. Cependant, les tailles obtenues avec les doses de 0 et $2,25 \mathrm{~kg}$ de $\mathrm{Mo} / \mathrm{ha}$ à la troisième période et celles obtenues aux autres périodes de fauche, n'ont pas présenté de différences significatives $(\mathrm{p}>0,05)$ quelle que soit la dose de fertilisation.

Effet comparé de l'enfouissement d'Arachis glabrata et de Desmodium intortum fertilisés au molybdène sur la biomasse de Brachiaria ruziziensis à différentes périodes de fauche

La biomasse de la plante a été significativement influencée $(\mathrm{p}<0,05)$ par les périodes de fauche, en fonction des doses de molybdène (Figure 3).

Toutefois, les valeurs de biomasses ont été variables d'un niveau de fertilisation à l'autre, d'un précédent cultural à l'autre et d'une période à l'autre. Ainsi, à la première période de fauche, les biomasses obtenues avec le précédent $D$. intortum ont été significativement $(\mathrm{p}<0,05)$ plus élevées que celles obtenues avec $A$. glabrata, pour les trois dernières doses de fertilisation. Pour la dose contrôle, les biomasses obtenues avec les deux 
précédents ont été comparables $(p>0,05)$. Les biomasses obtenues avec le précédent $A$. glabrata, à la deuxième période de fauche ont été significativement $(\mathrm{p}<0,05)$ plus élevées, quel que soit le niveau de fertilisation au molybdène. A la troisième période de fauche, les biomasses obtenues avec les deux précédents sont restées comparables $(\mathrm{p}>0,05)$ pour les doses de $0 ; 0,75$ et $1,50 \mathrm{~kg}$ de $\mathrm{Mo} / \mathrm{ha}$, avant que la biomasse obtenue avec le précédent $D$. intortum ne devienne significativement $(\mathrm{p}<0,05)$ plus élevée avec la dose de 2,25 kg de Mo/ha. Par contre, les biomasses obtenues aux deux dernières périodes de fauche sont restées comparables ( $p>0,05)$ quels que soient le précédent cultural et le niveau de fertilisation.

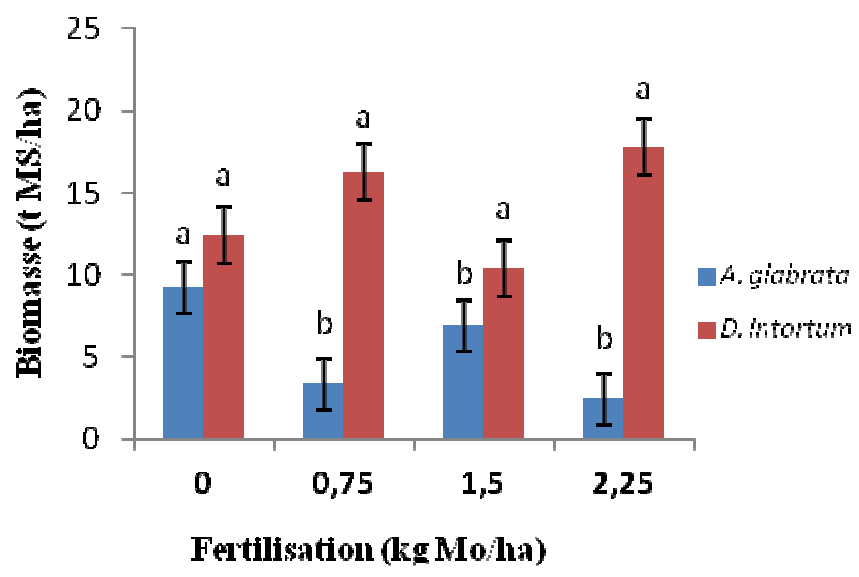

Figure 1: Evolution des biomasses enfouies des légumineuses en fonction des niveaux de fertilisation au molybdène. a,b : les moyennes portant les mêmes lettres pour le même niveau de fertilisation ne sont pas significativement différentes $(\mathrm{P}>0,05)$.

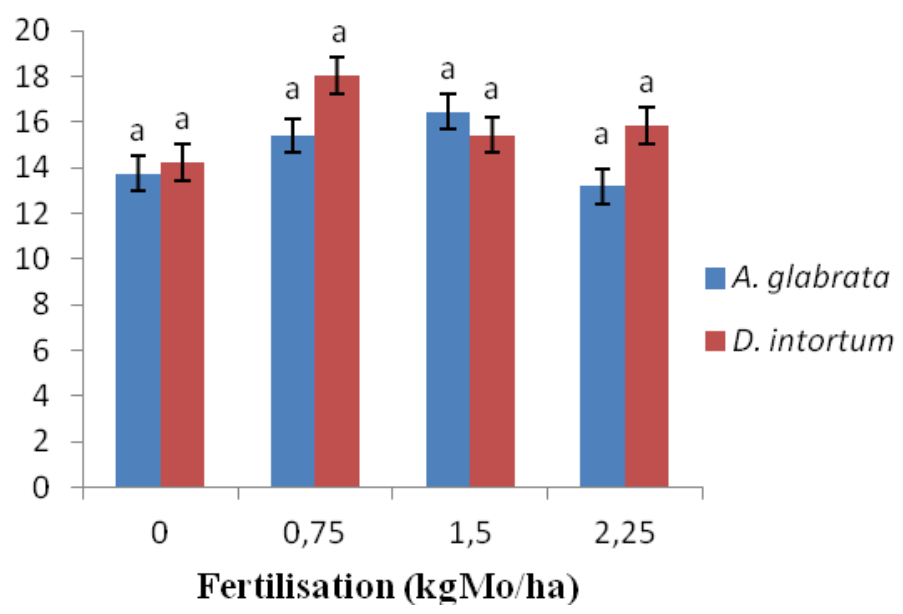

Figure 2: Effet de la fertilisation au molybdène sur les rapports carbone/azote $(\mathrm{C} / \mathrm{N})$ de $A$. glabrata et de $D$. intortum. 
Tableau 1: Evolution de la taille $(\mathrm{cm})$ des plantes de Brachiaria ruziziensis obtenue avec le précédent $A$. glabrata en fonction du niveau de fertilisation à différentes périodes de fauche.

\begin{tabular}{lcccccc}
\hline \multirow{2}{*}{$\begin{array}{c}\text { Niveau de Fertilisation } \\
\text { (kg Mo/ha) }\end{array}$} & \multicolumn{7}{c}{ Périodes de fauche* } \\
\cline { 2 - 7 } $\mathbf{0}$ & $\mathbf{P 1}$ & $\mathbf{P 2}$ & $\mathbf{P 3}$ & $\mathbf{P 4}$ & $\mathbf{P 5}$ & Moyennes \\
\hline $\mathbf{0 , 7 5}$ & 74,4 & 39,6 & 37,4 & 27,6 & 32,0 & 42,2 \\
$\mathbf{1 , 5}$ & 77,3 & 39,9 & 35,7 & 31,0 & 28,0 & 42,4 \\
$\mathbf{2 , 2 5}$ & 68,7 & 42,6 & 38,6 & 30,6 & 30,7 & 42,2 \\
SEM & 73,1 & 40,8 & 38,0 & 27,5 & 33,6 & 42,6 \\
Prob. & 1,93 & 0,63 & 0,66 & 0,86 & 0,93 & 3,7 \\
\hline
\end{tabular}

$*$ aucune différence significativement $(\mathrm{p}>0,05)$ a chaque période de fauche en fonction du niveau de fertilisation.

Tableau 2: Evolution de la biomasse (t MS/ha) de B. ruziziensis obtenue avec le précédent $A$. glabrata en fonction du niveau de fertilisation à différentes périodes de fauche.

\begin{tabular}{|c|c|c|c|c|c|c|c|}
\hline \multirow{2}{*}{$\begin{array}{l}\text { Niveau de Fertilisation } \\
\text { (kg Mo/ha) }\end{array}$} & \multicolumn{6}{|c|}{ Périodes de fauche } & \multirow[b]{2}{*}{$\begin{array}{l}\text { Total (t } \\
\text { MS/ha) }\end{array}$} \\
\hline & P1 & $\mathbf{P 2}$ & P3 & P4 & P5 & $\begin{array}{c}\text { Moyenne } \\
\text { s }\end{array}$ & \\
\hline $\mathbf{0}$ & $17,8^{\mathrm{a}}$ & $7,61^{b}$ & $4,51^{\mathrm{ab}}$ & $3,71^{\mathrm{a}}$ & $3,62^{\mathrm{a}}$ & $7,45^{\mathrm{a}}$ & 37,3 \\
\hline $\mathbf{0 , 7 5}$ & $18,7^{\mathrm{a}}$ & $9,63^{\mathrm{a}}$ & $3,90^{\mathrm{ab}}$ & $3,56^{\mathrm{a}}$ & $3,62^{\mathrm{a}}$ & $7,89^{\mathrm{a}}$ & 39,4 \\
\hline 1,5 & $16,9^{\mathrm{a}}$ & $7,14^{\mathrm{b}}$ & $4,62^{\mathrm{a}}$ & $3,48^{\mathrm{a}}$ & $4,05^{\mathrm{a}}$ & $7,24^{\mathrm{a}}$ & 36,2 \\
\hline 2,25 & $16,3^{\mathrm{a}}$ & $6,76^{\mathrm{b}}$ & $3,42^{\mathrm{b}}$ & $3,34^{\mathrm{a}}$ & $3,46^{\mathrm{a}}$ & $6,67^{\mathrm{a}}$ & 33,3 \\
\hline SEM & 0,47 & 0,41 & 0,21 & 0,12 & 0,11 & 1,23 & \\
\hline Prob. & 0,31 & 0,05 & 0,01 & 0,78 & 0,26 & 0,99 & \\
\hline
\end{tabular}

$\mathrm{a}, \mathrm{b}$ : les moyennes portant les mêmes lettres dans la même colonne pour la même fréquence ne sont pas significativement différentes $(\mathrm{P}>0,05) \cdot \mathrm{p} 1=\mathrm{p} 2=\mathrm{p} 3=\mathrm{p} 4=\mathrm{p} 5=$ période de fauche répétées de 45 jours.

Tableau 3: Evolution des tailles (cm) des plantes de Brachiaria ruziziensis obtenue avec le précédent $D$. intortum en fonction du niveau de fertilisation à différentes périodes de fauche.

\begin{tabular}{ccccccc}
\hline $\begin{array}{c}\text { Niveau de Fertilisation } \\
\text { (kg Mo/ha) }\end{array}$ & P1 & P2 & P3 & P4 & P5 & $\begin{array}{c}\text { Moyenne } \\
\text { S }\end{array}$ \\
\cline { 2 - 6 } & & & & & & $44,4^{\mathrm{a}}$ \\
$\mathbf{0}$ & $83,4^{\mathrm{a}}$ & $37,6^{\mathrm{a}}$ & $39,0^{\mathrm{a}}$ & $30,8^{\mathrm{a}}$ & $31,3^{\mathrm{a}}$ & $43,8^{\mathrm{a}}$ \\
$\mathbf{0 , 7 5}$ & $83,4^{\mathrm{a}}$ & $36,2^{\mathrm{a}}$ & $39,5^{\mathrm{a}}$ & $29,5^{\mathrm{a}}$ & $30,3^{\mathrm{a}}$ & $41,0^{\mathrm{a}}$ \\
$\mathbf{1 , 5}$ & $77,2^{\mathrm{a}}$ & $37,2^{\mathrm{a}}$ & $33,2^{\mathrm{b}}$ & $27,8^{\mathrm{a}}$ & $29,2^{\mathrm{a}}$ & $43,5^{\mathrm{a}}$ \\
$\mathbf{2 , 2 5}$ & $76,8^{\mathrm{a}}$ & $39,6^{\mathrm{a}}$ & $40,1^{\mathrm{a}}$ & $30,2^{\mathrm{a}}$ & $30,9^{\mathrm{a}}$ & 4,35 \\
SEM & 1,63 & 0,72 & 0,90 & 0,48 & 1,01 & 0,99 \\
Prob. & 0,29 & 0,42 & 0,04 & 0,14 & 0,91 & \\
\hline
\end{tabular}

$\mathrm{a}, \mathrm{b}$ : les moyennes portant les mêmes lettres dans la même colonne pour la même fréquence ne sont pas significativement différentes $(\mathrm{P}>0,05)$. 
Tableau 4: Evolution de la biomasse (t MS/ha) de Brachiaria ruziziensis obtenue avec le précédent D. intortum en fonction du niveau de fertilisation à différentes périodes de fauche.

\begin{tabular}{llllllll}
\hline & \multicolumn{7}{c}{ Périodes de fauche } \\
\hline $\begin{array}{l}\text { Niveau de Fertilisation } \\
\text { (kg Mo/ha) }\end{array}$ & P1 & P2 & P3 & P4 & P5 & Moyennes & Total \\
\hline $\mathbf{0}$ & & & & & & & \\
$\mathbf{0 , 7 5}$ & $22,28^{\mathrm{a}}$ & $5,52^{\mathrm{a}}$ & $6,26^{\mathrm{a}}$ & $3,26^{\mathrm{a}}$ & $4,33^{\mathrm{a}}$ & $8,33^{\mathrm{a}}$ & 41,65 \\
$\mathbf{1 , 5}$ & $24,99^{\mathrm{a}}$ & $5,63^{\mathrm{a}}$ & $5,93^{\mathrm{a}}$ & $3,34^{\mathrm{a}}$ & $4,33^{\mathrm{a}}$ & $8,84^{\mathrm{a}}$ & 44,22 \\
$\mathbf{2 , 2 5}$ & $22,43^{\mathrm{a}}$ & $5,75^{\mathrm{a}}$ & $6,02^{\mathrm{a}}$ & $3,41^{\mathrm{a}}$ & $4,49^{\mathrm{a}}$ & $8,42^{\mathrm{a}}$ & 42,10 \\
SEM & $20,63^{\mathrm{a}}$ & $5,05^{\mathrm{a}}$ & $6,07^{\mathrm{a}}$ & $3,41^{\mathrm{a}}$ & $4,14^{\mathrm{a}}$ & $7,86^{\mathrm{a}}$ & 39,30 \\
Prob. & 0,87 & 0,15 & 0,18 & 0,12 & 0,24 & 1,653 & \\
\hline
\end{tabular}

a : les moyennes portant les mêmes lettres dans la même colonne ne sont pas significativement différentes au seuil de $5 \%$.

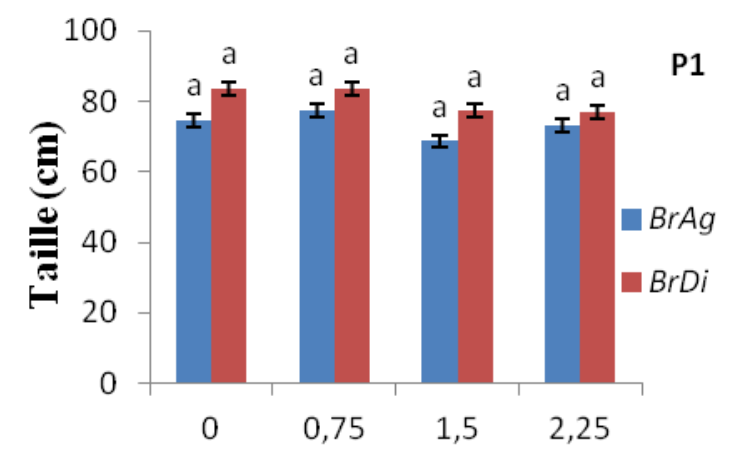

Fertilisation (kgMo/ha)
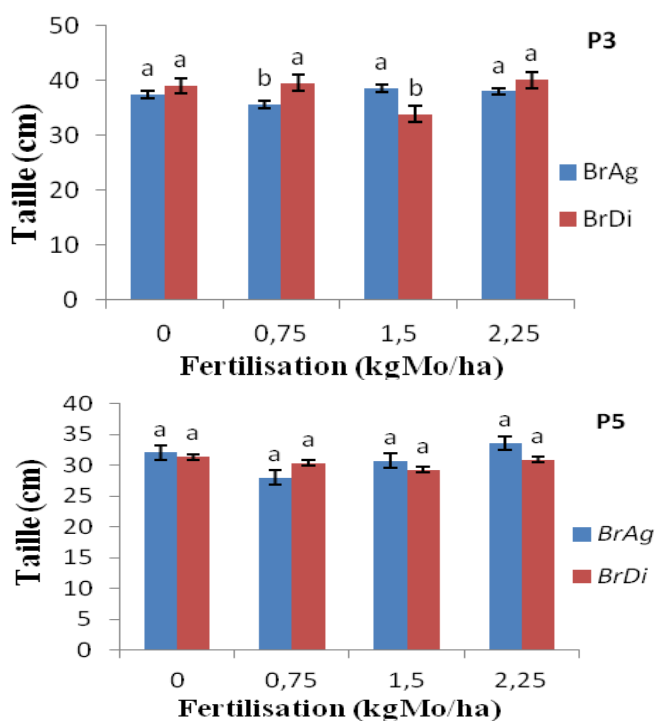
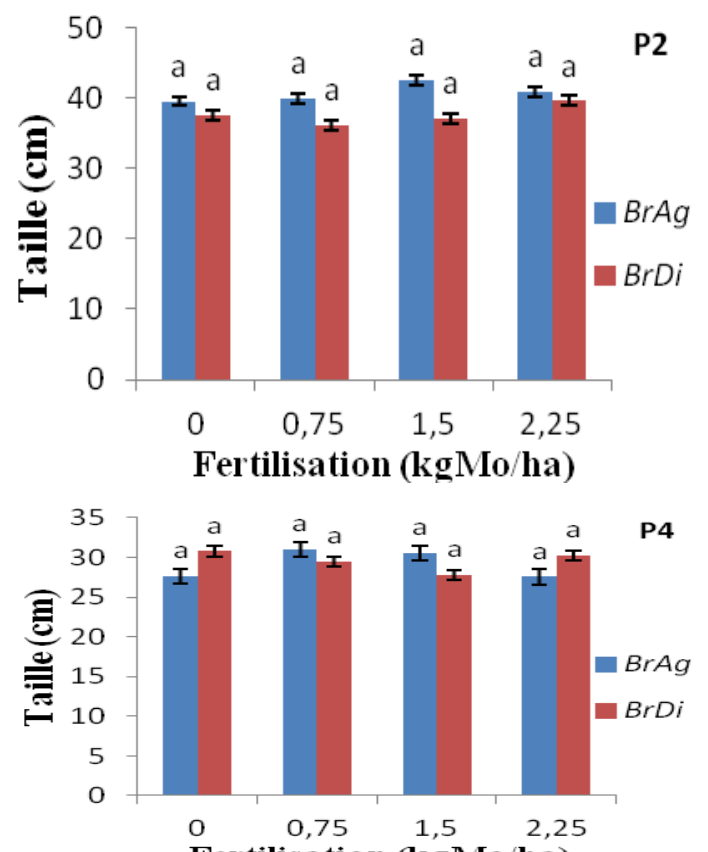

Fertilisation (kgMo/ha)
$\mathbf{P 1 = P 2 = P 3 = P 4 = P 5 = ~ P e ́ r i o d e ~ d e ~ f a u c h e ~ r e ́ p e ́ t e ́ e ~ d e ~} 45$ jours. BrAg= B. ruziziensis obtenu avec le précédent A. glabrata BrDi= B. ruziziensis obtenu avec le précédent D. intortum

Figure 2: Evolution comparée de la taille de Brachiaria ruziziensis en fonction des précédents A. glabrata et D. intortum fertilisés au molybdène à différentes périodes de fauche. 

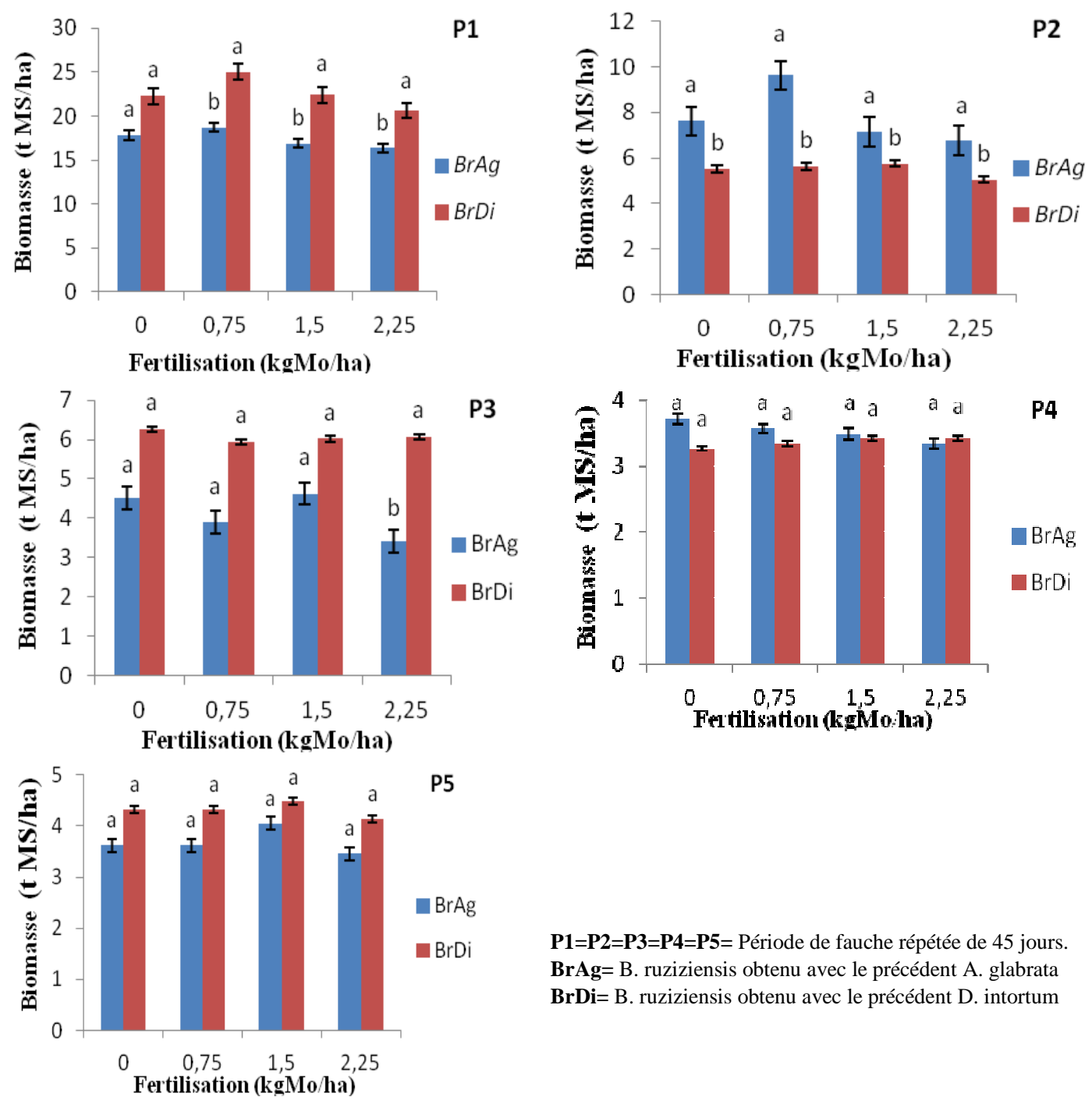

$\mathbf{P 1 =} \mathbf{P 2}=\mathbf{P 3}=\mathbf{P} 4=\mathbf{P 5}=$ Période de fauche répétée de 45 jours. BrAg= B. ruziziensis obtenu avec le précédent A. glabrata $\mathbf{B r D i}=\mathrm{B}$. ruziziensis obtenu avec le précédent D. intortum

Figure 3: Evolution comparée de la biomasse de Brachiaria ruziziensis en fonction des précédents A. glabrata et $D$. intortum fertilisés au molybdène à différentes périodes de fauche.

\section{DISCUSSION}

L'incorporation au sol de A. glabrata et de $D$. intortum, fertilisés au molybdène a influencé de manière variable la taille des plantes de $B$. ruziziensis aux différentes périodes de fauche. Cette situation traduit le faible effet qu'aurait eu le molybdène sur la nodulation des légumineuses. En effet, le molybdène étant un constituant des enzymes impliquées dans la fixation de l'azote gazeux par les Rhizobiums des légumineuses, devrait permettre ainsi de reconstituer les réserves des matières organiques et ajouter des matériaux azotés au sol (Skerman, 1982), pour assurer la croissance de la plante. Mais malheureusement, cela a été très peu observé dans le cadre de cette étude. Ce résultat corrobore les résultats des travaux de Jongruaysup et al. (1993) qui a observé que le molybdène n'influence pas la biomasse nodulaire des légumineuses. Cependant, les tailles les plus élevées ont été observées à la première période de fauche avec la dose de $0,75 \mathrm{~kg}$ de $\mathrm{Mo} / \mathrm{ha}$, pour les deux précédents 
légumineuses. Ainsi donc, les résultats sur les tailles de B. ruziziensis observés dans cette étude, seraient liés à l'effet combiné de l'azote apporté au sol par le biais de la fixation symbiotique et celui de l'azote apporté par le rythme de dégradation des racines et des biomasses des légumineuses enfouies. En effet, les exsudats racinaires des légumineuses laissées sur place, seraient capables de solubiliser les phosphates de calcium et le phosphore occlue, augmentant ainsi la disponibilité du phosphore assimilable et son absorption par la culture subséquente (Alvey et al., 2001). De ce fait B. ruziziensis, comme toutes les plantes, exige pour sa croissance, des éléments minéraux qu'il puise dans le sol. La matière organique ainsi fournie par l'apport de la biomasse végétale, intervient dans les mécanismes de la libération de l'azote minéral, donc de la nutrition de la plante (Rouanet, 1984). Selon le même auteur, la biomasse végétale enfouie contiendrait au moins cinq (5) éléments minéraux majeurs à savoir l'azote $(\mathrm{N})$, le calcium $(\mathrm{Ca})$, le magnésium $(\mathrm{Mg})$, le potassium $(\mathrm{K})$, le soufre (S) et le phosphore (P) qui, mis à la disposition de la plante, peuvent influencer considérablement sa croissance.

Les tailles comparées de B. ruziziensis obtenues avec les précédents culturaux en fonction des doses de molybdène n'ont été significativement $(\mathrm{p}<0,05)$ influencées qu'à la troisième période de fauche. Alors qu'aux deux premières et aux deux dernières périodes, les tailles n'ont pas été significativement différentes. Cette observation pourrait s'expliquer par la dynamique de minéralisation des légumineuses enfouies. En effet, aux deux premières périodes, les légumineuses n'avaient pas encore libéré assez d'éléments nutritifs nécessaires à la croissance de $B$. ruziziensis. Aux deux dernières périodes, on pourrait penser probablement dans ces conditions, à une baisse de l'effet résiduel des légumineuses dans le temps. Ainsi, les valeurs de tailles observées à ces périodes seraient dues essentiellement à la réserve en nutriments du sol. Et du coup, la croissance de B. ruziziensis se trouve ralentie. Les tailles ont été variables d'un précédent à l'autre, d'un niveau de fertilisation à l'autre et d'une période de fauche à l'autre. Toutefois, dans l'ensemble, $D$. intortum a produit les plantes de $B$. ruziziensis avec la taille la plus élevée $(83,43 \mathrm{~cm})$ par rapport à celle obtenue avec $A$. glabrata $(77,29 \mathrm{~cm})$ pour la même dose de $0,75 \mathrm{~kg}$ de $\mathrm{Mo} / \mathrm{ha}$ et à la première période de fauche. Cette différence de taille de la plante subséquente en fonction du précédent cultural, serait certainement attribuable à la quantité de biomasse enfouie, à la composition chimique de celle-ci, du rapport $\mathrm{C} / \mathrm{N}$ et au rythme de dégradation des résidus. En effet dans cette étude, la biomasse enfouie du précédent $D$. intortum était plus élevée que celle de $A$. glabrata. Cette observation rejoint celle de Wani et al. (1995) qui attribue la variabilité de la productivité de la culture subséquente à la qualité et à la quantité des espèces enfouies. D'après le même auteur, l'efficacité de l'action de l'enfouissement dépend de la nature du résidu (rapport $\mathrm{C} / \mathrm{N}$, proportions des composés solubles, de lignine, de cellulose et hémicellulose) et à la disponibilité en minéraux du sol. De même, les résultats obtenus par Boukila et al. (2009) ont montré que l'azote résiduel de $D$. intortum $(1,67$ $\% \mathrm{MS})$ était plus élevé que celui obtenu avec A. glabrata $(0,57 \% \mathrm{MS})$ et A. glabrata était plus riche en cellulose que $D$. intortum, ce qui aurait pour effet de retarder la minéralisation de A. glabrata.

L'enfouissement des deux précédents culturaux fertilisés au molybdène a relativement influencé les biomasses de $B$. ruziziensis. Ce résultat ne corrobore pas ceux observés par de nombreux auteurs (Paillat et al., 1999; Obulbiga et Kaboré-Zoungrana, 2007; Pamo et al., 2008; Tendonkeng et al., 2009) qui ont montré que la biomasse des fourrages était significativement influencée par la fertilisation azotée. La différence d'effet, serait donc liée au type de la plante enfouie et surtout à sa dynamique de mise à la disposition de la plante subséquente, des 
éléments minéraux nécessaires à sa production. Néanmoins, de manière générale, la biomasse la plus élevée (24,99 t MS/ha), obtenue avec le précédent $D$. intortum fertilisé à la dose de $0,75 \mathrm{~kg}$ de $\mathrm{Mo} / \mathrm{ha}$, à la première période de fauche, est inférieure à celle obtenue (26,5 t MS/ha) par Olsen (1982) avec B. ruziziensis en Ouganda. Mais elle est cependant supérieure à celle obtenue $(13,24 \mathrm{t}$ MS/ha) par Tendonkeng et al. (2009) avec la dose de fertilisation de $200 \mathrm{~kg} \mathrm{~N} /$ ha sur le même fourrage, au même stade phénologique et dans la même zone. Cet écart de production en faveur de l'enfouissement, serait attribuable au fait qu'au-delà de son rôle important dans la fourniture du sol en azote, l'enfouissement influe, selon sa nature, sur les propriétés physico-chimiques et biologiques du sol et à la capacité d'emmagasiner les réserves en eau (Rouanet, 1984). Ce qui aurait pour conséquence, l'amélioration des conditions nutritionnelles de la plante.

Lorsque l'on compare les biomasses de B. ruziziensis obtenues en fonction du rythme de fauche et des précédents légumineuses, on constate que les biomasses ont varié de manière irrégulière d'un niveau de fertilisation à l'autre. Toutefois, la biomasse la plus élevée a été obtenue avec le précédent $D$. intortum à la première période de fauche. Cette irrégularité dans la production du fourrage avec le précédent cultural dans le temps, s'expliquerait par la dynamique de minéralisation qui est fonction de la nature du résidu et surtout de l'effet de la défoliation, à l'origine de la baisse de l'absorption d'azote minéral $\left(\mathrm{NH}_{4}^{+}\right.$ou $\left.\mathrm{NO}_{3}^{-}\right)$. Dès lors, les réserves des organes pérennes semblent jouer un rôle essentiel pour la fourniture d'azote ou du carbone aux parties aériennes en repousse (Cliquet et al., 1990). Ainsi, les plantes fourragères symbiotiques ou non, sont donc confrontées en début de repousse à une réduction de leur capacité à assimiler l'azote minéral. La néoformation d'organe ne pourra se réaliser, au moins dans un premier temps, qu'aux dépens des réserves de la plante en azote.
Les résultats de cette étude ont montré également que la biomasse de $B$. ruziziensis a baissé avec la période de fauche. Les biomasses les plus importantes ont été observées au deux premières périodes. Cela pourrait être lié à la qualité des amendements organiques (faibles rapports $\mathrm{C} / \mathrm{N}$ ) des deux légumineuses enfouies. En effet, ces rapports sont inférieures à 20, indiquant ainsi une minéralisation rapide des résidus (Coleman et al., 1989). Ces résultats suggéreraient que pour une rotation de culture avec enfouissement de $A$. glabrata et de $D$. intortum, le rythme d'exploitation de $B$. ruziziensis avec 45 jours de repos, se limiterait à deux périodes de fauche. Cette observation est deux fois moins que celle proposée par Phillips et al. (1983) qui a estimé ce rythme à quatre cycles successifs de repousse de fourrage. La baisse de biomasse et l'absence de différences significatives enregistrées aux trois dernières fréquences de fauche, aurait donc pour explication, le rôle joué par les réserves azotées dans la dynamique de repousse. Ainsi, la disponibilité en réserves azotée au moment de la coupe est essentielle à l'élaboration d'une nouvelle biomasse. En effet, plus le niveau de réserve azotée est élevé, plus leur mobilisation, l'absorption d'azote et en conséquence la croissance des parties aériennes sont importantes (Ourry et al., 1994).

\section{Conclusion}

$\mathrm{Au}$ terme de cette étude, il ressort que l'enfouissement d'Arachis glabrata et de Desmodium intortum fertilisés au molybdène a:

- influencé de manière variable la taille des plantes de Brachiaria ruziziensis aux différentes périodes de fauche. Dans l'ensemble, Desmodium intortum a induit la taille la plus élevée $(83,43 \mathrm{~cm})$ par rapport à celle obtenue avec Arachis glabrata (77,29 $\mathrm{cm})$ pour la même dose de $0,75 \mathrm{~kg}$ de $\mathrm{Mo} / \mathrm{ha}$, à la première période de fauche.

- relativement influencé la biomasse de Brachiaria ruziziensis. La valeur de la 
biomasse la plus élevée (24,99 t MS/ha) étant obtenue avec la dose de $0,75 \mathrm{~kg}$ de $\mathrm{Mo} / \mathrm{ha}$ sous le précédent Desmodium intortum à la première période de fauche.

Les résultats de cette étude suggéreraient que pour une rotation de culture avec enfouissement d'Arachis glabrata et de Desmodium intortum, le rythme d'exploitation de Brachiaria ruziziensis avec 45 jours de repos, se limiterait à deux (2) périodes de fauche. Aussi, l'enfouissement de D. intortum fertilisé à $0,75 \mathrm{~kg}$ de $\mathrm{Mo} / \mathrm{ha}$, peut constituer l'engrais vert à recommander dans la pratique pour la culture de B. ruziziensis dans ces conditions. Par ailleurs, il serait souhaitable que cette étude se poursuive pour déterminer avec précision:

- le stade de croissance le plus approprié pour l'enfouissement de cette légumineuse. En effet, de ces stades, dépendent la composition chimique de la plante en générale et sa teneur en azote en particulier, ce qui influence sa contribution en azote du sol;

- le moment de séparation idéal entre l'incorporation de la légumineuse au sol et la mise en place des cultures subséquentes pour faire coïncider les besoins des plantes suivantes à la libération au sol des éléments nutritifs par le processus de la décomposition de la légumineuses.

\section{REMERCIEMENTS}

Les auteurs remercient l'Université de Dschang pour son appui académique et logistique et le Programme d'Appui Institutionnel - Développement des Ressources Humaines (PAI-DRH) du Ministère du Budget, des Comptes Publics et de la Fonction publique du Gabon pour son apport financier à la réalisation de ce travail.

\section{REFERENCES}

Akedrin TN, N'guessan K, Ake-assi E, Ake S. 2010. Effet de légumineuses herbacées ou subligneuses sur la productivité du maïs. Journal of Animal and Plant Sciences, 8(2): 953-963.

Alvey S, Bagayoko M, Neumann G, Buerkert A. 2001. Cereal/legume rotation chemical properties and biological activities in two
West African soils. Plant and Soil, 231: 45-54.

Boukila B, Tendonkeng F, Pamo ET, Betfiang ME. 2009. Composition chimique et digestibilité in vitro de Desmodium incinatum, Desmodium intortum et Arachis glabrata incubés seuls ou mélangés avec des chaumes de maïs. Livestock Research for Rural Development. 21, Article \#108. Retrieved August 28, 2009, from http//www. Irrd.org/Irrd21/7/bouk21108.htm.

Brkić S, Milakovic Z, Kristek A, Antunovi M. 2004. Pea yield and its quality depending on inoculation, nitrogen and molybdenum fertilization. Plant, Soil and Environment, 39(1): 39-45.

Cliquet JB, Deléens E, Mariotti A. 1990. C and $\mathrm{N}$ mobilization from stalk and leaves during kernel filling by $\mathrm{C}$ and $\mathrm{N}$ tracing in Zea mays L. Plant Physiology, 94: 1547-1553.

Coleman DC, Malcom O, Goro U. 1989. Dynamic of soil organic mater. In Interaction of Soil Organic Matter and Variable-Charges Clays; Dynamic of Soil Organic Mater in Tropical Ecosystems. Malcolm $\mathrm{O}$ et al. (eds), University of Hawaii.

Frankenberger WTjr, Abdelmagid HM. 1985. Kinetic parameters of nitrogen mineralization rate of leguminous crops incorporated into soil. Plan and Soil, 87: 257-271.

Janzen HH, Bole JB, Biederberk VO, Slinkard AE. 1990. Fate of $\mathrm{N}$ applied as green manure or ammonium fertilizer to soil subsequently cropped with spring wheat at three sites in Western Canada. Canadian Journal of Soil Sciences, 70: 313-323.

Jongruaysup S, O’Hara GW, Dell B, Bell RW. 1993. Effet de la fertilisation au molybdèneafaible doses sur l'initiation des nodules, le développement et la fixation de $\mathrm{N}_{2}$ sur noir gramme (vigna mungo L.). Plant and Soil, 155-156(1) : 345-348.

Le soudeur C, Labreuche J. 2007. Implanter une légumineuse à l'interculture. Perspectives Agricoles. http://www. agriculture-de-conservation.com/IMG/ pdf/PA-2007-04-legCI-pdf 
Mahler RL, Hemanda H. 1993. Evaluation of nitrogen fertilizer value of plants materials to spring wheat production. Agronomy Journal, 85: 305-309.

Morot-Gaudry JF. 1997. Assimilation de l'Azote Chez les Plantes. Aspects Physiologique, Biochimique et Moléculaire. INRA : Paris ; 422p.

Obulbiga MF, Kaboré-Zoungrana CY. 2007. Influence de la fumure azotée et du rythme d'exploitation sur la production de matière sèche et la valeur alimentaire d'Andropogon gayanus kunth au Burkina Faso. Tropicultura, 25(3): 161-167.

Olse FJ. 1982. Effect of large application of nitrogen fertilizer on the production and protein contents of four tropical grasses in Uganda. Tropical Agriculture, 49: 251260.

Ourry A, Kim TH, Boucaud J. 1994. Nitrogen reserves mobilization during regrowth of Medicago sativa L.: Relationships between their availability and regrowth yield. Plant Physiology, 105, 831-837.

Paillat JM, Rippstein G, Huguenin J, Marnotte P, Déat M. 1999. Etablissement et entretien des prairies. In Cultures Fourragères Tropicale, Roberge G, Toutain B (eds). CIRAD; 215-247.

Pamo TE, Boukila B, Meduke CN, Tendonkeng F. 2008. Effect of nitrogen fertilisation and cutting frequency on the yield and regrowth of Panicum maximum Jacq in west Cameroon. In XXI International Grassland Congress / VIII International Rangeland Congress Hohhot, Xie Haining, Huang Jiehua (eds). China, $29^{\text {th }}$ June $-5^{\text {th }}$ July $2008,354$.

Pauwels JM, Van Ranst E, Verloo M, Mvondo Ze A. 1992. Méthode d'analyse de sols et de plantes, gestion de stock de verrerie et de produits chimiques. Manuel de Laboratoire de Pédologie. Publications Agricoles $\mathrm{N}^{\circ} \mathbf{2 8}$.

Phillips DA, Center DM, Jones MB. 1983. Nitrogen turnover and assimilation during regrowth in trifolium subterraneum $\mathrm{L}$. And Bronus mollis L. Plant Physiology, 71: 472-476.

Roberge G, Toutain B. 1999. Choix des Cultures Fourragères Tropicales, Roberge G, Toutain B (eds). Cirad: Montpellier, France ; 321-357

Rouanet G. 1984. Le Maïs : Technicien d'Agriculture Tropicale. Agence de Coopération Culturelle et Technique. Edn. Maisonneuve et Larose: Paris. $142 p$.

Segda Z, Hien V, Lompo F, Becker M. 1998. Gestion améliorée de la jachère par l'utilisation de légumineuses de couverture. In Plantes de Couverture en Afrique de l'Ouest. Une Contribution à l'Agriculture Durable, Buckles D, Etèka A, Osiname O, Galiba M, Galiano N (eds). CIEPCA ; 318p.

Skerman PJ. 1982. Les Légumineuses Fourragères Tropicales. Collection FAO: Production Végétale et Protection des Plantes. FAO : Rome, Italie ; 666p.

Tendonkeng F, Boukila B, Pamo TE, Mboko AV, Matumuini NEF, Fogang ZB. 2009. Effet de différents niveaux de fertilisation azotée et du stade phénologique sur la croissance et le rendement de Brachiaria ruziziensis dans l'Ouest Cameroun. International Journal of Biological and Chemical Sciences, 4(4): 725-735.

Wani S, Rupela OP, Lee KK. 1995. Sustainable agriculture in the semi-arid tropics through biological nitrogen fixation in grain legumes. Plant and Soil, 174: $29-49$. 